\title{
Rotating Variable-Thickness Inhomogeneous Cylinders: Part I-Analytical Elastic Solutions
}

\author{
Ashraf M. Zenkour \\ Department of Mathematics, Faculty of Science, King AbdulAziz University, Jeddah, Saudi Arabia \\ Department of Mathematics, Faculty of Science, Kafrelsheikh University, Egypt \\ E-mail: zenkour@gmail.com \\ Received June 2, 2010; revised December 12, 2010; accepted December 16, 2010
}

\begin{abstract}
In this paper, an analytical solution for the rotation problem of an inhomogeneous hollow cylinder with variable thickness under plane strain assumption is developed. The present cylinder is made of a fiberreinforced viscoelastic inhomogeneous orthotropic material. The thickness of the cylinder is taken as parabolic function in the radial direction. The elastic properties varies in the same manner as the thickness of the cylinder while the density varies according to an exponential law form. The inner and outer surfaces of the cylinder are considered to have combinations of free and clamped boundary conditions. Analytical solutions are given according to different types of the hollow cylinders. An extension of the present solutions to the viscoelastic ones and some applications are investigated in Part II.
\end{abstract}

Keywords: Rotating, Inhomogeneous Cylinders, Orthotropic, Variable Thickness and Density

\section{Introduction}

The rotation problem of inhomogeneous cylinder has been important applications, particularly in mechanical engineering, aerospace industry, underwater vehicles and biomechanics. The pertinent literature on the investiga tion of stresses and displacements in an inhomogeneous hollow circular cylinder may be reviewed here. The plane strain problem of a rotating inhomogeneous orthotropic hollow cylinder is solved by Senitskii [1]. Horgan and Chan [2] analyzed two-dimensional plane stress/strain deformations by assuming Youngs modulus to be a power law function of the radial direction of the cylinder and constant Poisson's ratio. Vasilenko and Klimenko, [3] have analyzed the stress state of a rotating cylinder, inhomogeneous in the radial direction, having one plane of elastic symmetry and loaded with centrifugal forces. Rooney and Ferrari [4] have examined the tension, bending, and flexure of cylinders with functionally graded (FG) cross-section. The effect of inhomogeneity of elastic properties and density in the circumferential direction on the distribution of stress and displacement in orthotropic cylindrical panels using load in the axial direction is investigated by Grigorenko and Vasilenko [5]. Oral and Anlas [6] have analyzed the effect of continuous inhomogeneity on the stress distribution in an anisotropic cylinder. Pan and Roy [7] have solved a plane-strain problem for a FG cylinder by dividing it into several homogeneous cylinders. Tutuncu [8] has gave the power series solution for stresses and displacements in FG cylinders with exponentially-varying elastic modulus through the radial direction. Theotokoglou and Stampouloglou [9] have studied axisymmetric problems for radially inhomogeneous circular cylinders. The effect of varying Poisson's ratio on deformation fields in FG cylinders has been investigated by Mohammadi and Dryden [10]. Li and Peng [11] have analyzed axisymmetric deformations of FG hollow cylinders and disks with arbitrarily varying material properties.

In recent years considerable attention has been given to solutions for the cylinders with variable thickness. Variable-thickness hollow cylinder is a common structure type which can be used in some applications involving aerospace, submarine structures, nuclear reactors as well as chemical pipes. Grigorenko and Rozhok [12] have studied the stress problem for non-circular hollow cylinder with variable thickness under uniform and local loads. Zenkour [13] has established the stresses in a rotating variable-thickness orthotropic cylinder containing a solid core of uniform-thickness. Also, Zenkour [14] has analytically investigated the behavior of composite circular cylinders subjected to internal and ex- 
ternal surface loading. The cylinder consists of a number of homogeneous ply groups of axially variable thickness. Duan and Koh [15] have derived analytical solutions for axisymmetric transverse vibration of cylindrical shells with thickness varying monotonically in arbitrary power form due to forces acting in the transverse direction. Nie and Batra [16] have studied plane-strain static deformations of a cylinder with elliptical inner and circular outer surfaces composed of a material that is polar-orthotropic and its moduli vary exponentially in the radial direction.

In this paper, the rotating fiber-reinforced viscoelastic hollow cylinder is analytically studied. The thickness of the cylinder, the elastic properties and density are taken to be functions in the radial coordinate. The governing second-order differential equation is derived and solved with the aid of some hypergeometric functions. The displacement and stresses for rotating variable-thickness inhomogeneous orthotropic hollow cylinder subjected to various boundary conditions are obtained. Special cases of the studied problem are established.

\section{Formulation of the Problem}

Consider an elastic hollow cylinder made of an inhomo geneous, orthotropic material and rotates about its axis. The cylindrical coordinates $(r, \theta, z)$ are chosen such that the axial coordinate $z$ coinciding with the axis of rotation, $r$ is the radial coordinate. Assuming the cylinder is symmetric with respect to the $z$-axis, we have only the radial displacement $u$ which is independent of the circumferential coordinate $\theta$. Furthermore, in the planes perpendicular to the $z$-axis in plane strain, $u$ is a function of $r$ alone. Consequently, the Cauchy's relations under considerations can be written in the following form:

$$
\varepsilon_{r r}=\frac{d u}{d r}, \quad \varepsilon_{\theta \theta}=\frac{u}{r}, \quad \varepsilon_{z z}=\varepsilon_{r \theta}=\varepsilon_{r z}=\varepsilon_{\theta z}=0,
$$

where $\varepsilon_{i j}$ are the strain components.

From the generalized Hooke's law and using the above geometric relations, we can obtain the stress components for an orthotropic cylinder in the following form:

$$
\begin{aligned}
& \sigma_{r r}=c_{11} \frac{d u}{d r}+c_{12} \frac{u}{r}, \quad \sigma_{\theta \theta}=c_{12} \frac{d u}{d r}+c_{22} \frac{u}{r} \\
& \sigma_{z z}=c_{13} \frac{d u}{d r}+c_{23} \frac{u}{r}, \quad \sigma_{r \theta}=\sigma_{r z}=\sigma_{\theta z}=0
\end{aligned}
$$

where $c_{i j}$ are the elastic properties. Let us assume now that the thickness $h$ of the cylinder varies in the radial direction in a parabolic form given by:

$$
h(r)=h_{0}\left[1-n(r / b)^{k}\right], \quad 0 \leq n<1, k>0,
$$

where $h_{0}$ is the thickness at the axis of the cylinder, $n$ and $k$ are geometric parameters and $b$ is the external radius of the cylinder. The parameter $k$ determines the shape of the thickness profile while $n$ determines the thickness at the surface of the cylinder relative to $h_{0}$. For three sets of geometric parameters $n$ and $k$, the dimensionless thickness $h / h_{0}$ as a function of the dimensionless radius $r / b$ is described by the profiles shown in Figure 1 for $b=5 a$ in which $a$ is the inner radius of the cylinder. In Figure 1(a) the thickness profile is concave for $k<1$ while in Figures. 1(b) and 1(c) it is convex for $k>1$. Furthermore, the thickness of the cylinder is linearly decreasing by setting $k=1$.

As the effect of thickness variation of rotating cylinders can be taken into account in their equilibrium equation, the theory of the cylinders of variable thickness can give excellent results as that of the uniform thickness
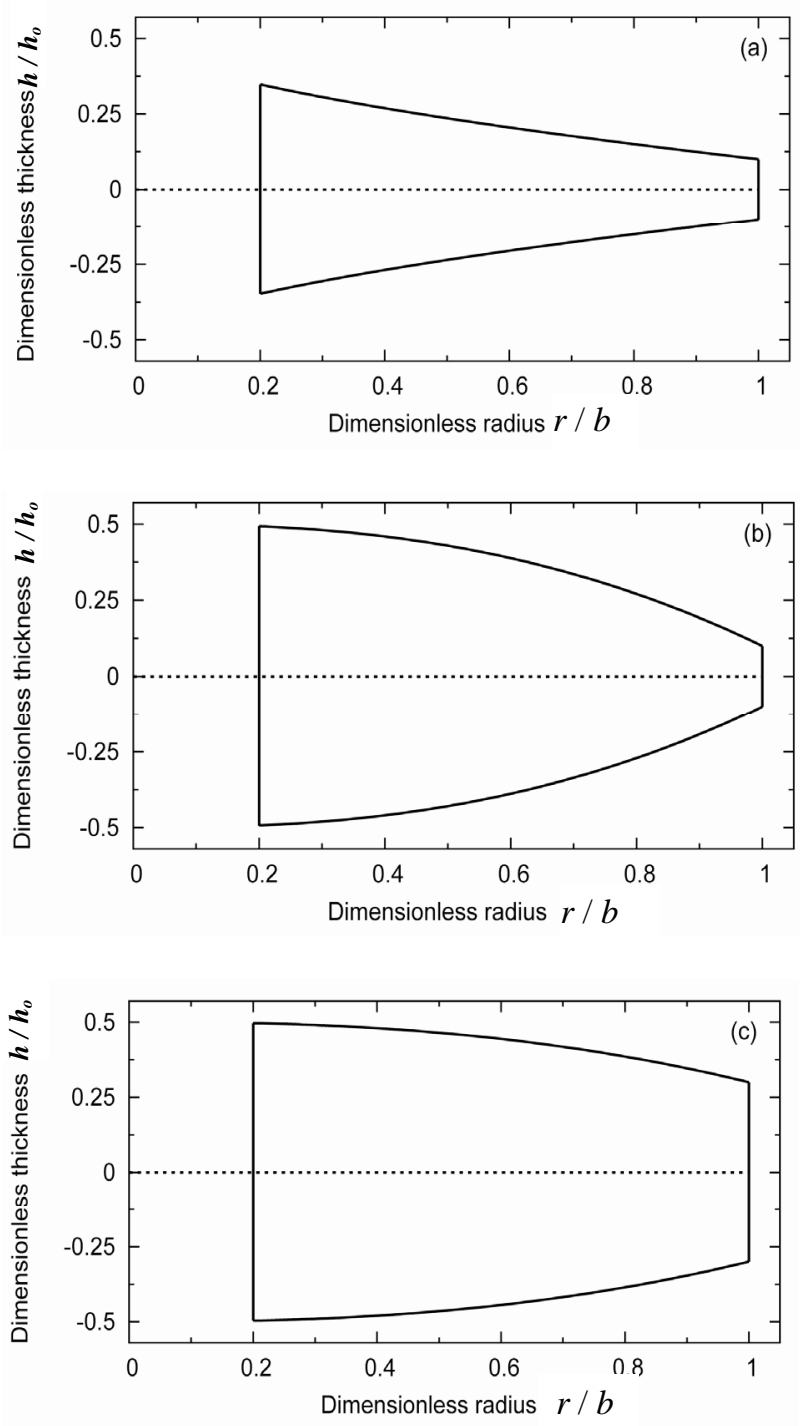

Figure 1. Parabolic cylinder profiles: (a) $k=0.6 ; n=0.8$; (b) $\mathrm{k}=2.5 ; \mathrm{n}=0.8 ;$ (c) $\mathrm{k}=2.5 ; \mathrm{n}=0.4$. 
cylinders as long as they meet the assumption of plane strain. After considering this effect, the equilibrium equation of rotating cylinder with variable thickness can be written as:

$$
\frac{d}{d r}\left(h r \sigma_{r r}\right)-h \sigma_{\theta \theta}+h \rho \Omega^{2} r^{2}=0,
$$

where $\Omega$ is the constant angular velocity and $\rho$ is the density of the cylinder material.

We characterize the elastic properties $c_{i j}$ and the material density $\rho$ of inhomogeneous cylinder by:

$$
\begin{array}{r}
c_{i j}=\alpha_{i j}\left[1-n(r / b)^{k}\right], \rho=\rho_{0} e^{-m(r / b)^{k}}, \quad \text { (5) for the radial displacement } u(r): \\
r^{2} \frac{d^{2} u}{d r^{2}}+r\left[1-\frac{2 n k(r / b)^{k}}{1-n(r / b)^{k}}\right] \frac{d u}{d r}-\left[\lambda^{2}+\frac{2 n \mu k(r / b)^{k}}{1-n(r / b)^{k}}\right] u+\frac{\rho_{0} \Omega^{2} r^{3} e^{-m(r / b)^{k}}}{\alpha_{11}\left[1-n(r / b)^{k}\right]}=0,
\end{array}
$$

where

$$
\lambda=\sqrt{\alpha_{22} / \alpha_{11}}, \mu=\alpha_{12} / \alpha_{11} .
$$

Introducing the dimensionless radius $\bar{r}=r / b$ in Equation (6), then its general solution can be written as

$$
\begin{aligned}
u(\bar{r})=n^{(1 / 2+\lambda / k)} \hat{C}_{1} P_{1}(\bar{r})+n^{(1 / 2-\lambda / k)} \hat{C}_{2} P_{2}(\bar{r})+R(\bar{r}), \quad \text { (8) in which } \\
i=1+\frac{\lambda-\ell}{k}, \quad j=1+\frac{\lambda+\ell}{k}, \quad \delta=1+\frac{2 \lambda}{k}, \quad \ell=\sqrt{k^{2}+\lambda^{2}-2 \mu k .}
\end{aligned}
$$

Note that, the function $M(\xi, \eta, \zeta, z)$ is the generalized hypergeometric function defined by [17]:

$$
M(\xi, \eta, \zeta, z)=\sum_{q=0}^{\infty} \frac{\xi_{q} \eta_{q} z^{q}}{\zeta_{q} q !}, z=n \bar{r}^{k},
$$

where $\xi_{q}$, for example, is the Pochhammer's symbol given by

$$
\xi_{q}=\xi(\xi+1)(\xi+2) \cdots(\xi+q-1)=\frac{\Gamma(\xi+q)}{\Gamma(\xi)},
$$

in which $\Gamma$ represents Gamma function. It is to be noted that, for real values of the upper parameters $\xi$ and $\eta$, and non-zero real value of the lower parameter $\zeta$ the generalized hypergeometric function $M(\xi, \eta, \zeta, z)$ converges for $|z|<1$.

The particular solution $R(\bar{r})$ for Equation (8) is obtained using variation of parameters as

$$
R(\bar{r})=U_{1}(\bar{r}) P_{1}(\bar{r})+U_{2}(\bar{r}) P_{2}(\bar{r}),
$$

where

$$
U_{1}(\bar{r})=-\int_{0}^{\bar{r}} \frac{P_{2}(\varphi) f(\varphi)}{\Delta(\varphi)} d \varphi, U_{2}(\bar{r})=\int_{0}^{\bar{r}} \frac{P_{1}(\varphi) f(\varphi)}{\Delta(\varphi)} d \varphi,
$$

in which

$$
f(\bar{r})=-\frac{\rho_{0} \Omega^{2} b^{3} \bar{r} e^{-m \bar{r}^{k}}}{\alpha_{11}\left(1-n \bar{r}^{k}\right)},
$$

and $\Delta(\bar{r})$ is the Wronskian given by

$$
\Delta(\bar{r})=P_{1}(\bar{r}) \frac{d P_{2}(\bar{r})}{d \bar{r}}-P_{2}(\bar{r}) \frac{d P_{1}(\bar{r})}{d \bar{r}} .
$$

Therefore,

$$
R(\bar{r})=\frac{\rho_{0} \Omega^{2} b^{3}}{\alpha_{11}}\left[\bar{r}^{(\lambda)} M_{1}(\bar{r}) \int_{0}^{\bar{r}} \frac{\varphi^{2-\lambda} e^{-m \varphi^{k}} M_{2}(\varphi)}{\left(1-n \varphi^{k}\right) F(\varphi)} d \varphi-\bar{r}^{(-\lambda)} M_{2}(\bar{r}) \int_{0}^{\bar{r}} \frac{\varphi^{2+\lambda} e^{-m \varphi^{k}} M_{1}(\varphi)}{\left(1-n \varphi^{k}\right) F(\varphi)} d \varphi\right]
$$

where

$$
F(\bar{r})=\bar{r}\left(M_{1}(\bar{r}) \frac{d M_{2}(\bar{r})}{d \bar{r}}-M_{2}(\bar{r}) \frac{d M_{1}(\bar{r})}{d \bar{r}}\right)-2 \lambda M_{1}(\bar{r}) M_{2}(\bar{r}) .
$$

Note that, the first derivative of the general hypergeometric function is given by:

$$
\frac{d}{d \bar{r}} M(\xi, \eta, \zeta, z)=\frac{\xi \eta d z}{\zeta d \bar{r}} M(\xi+1, \eta+1, \zeta+1, z) .
$$


Consequently, the exact general solution for the radial displacement can be written as

$$
u(\bar{r})=\bar{r}^{(\lambda)} M_{1}(\bar{r})\left[C_{1}+F_{1}(\bar{r})\right]+\bar{r}^{(-\lambda)} M_{2}(\bar{r})\left[C_{2}-F_{2}(\bar{r})\right],
$$

where

$$
C_{1}=n^{(1 / 2+\lambda / k)} \hat{C}_{1}, \quad C_{2}=n^{(1 / 2-\lambda / k)} \hat{C}_{2},
$$

and

$$
\begin{aligned}
& \sigma_{r r}(\bar{r})= \\
& \frac{1-n \bar{r}^{k}}{b}\left[\bar{r}^{(\lambda)}\left\{\left(C_{1}+F_{1}\right)\left(\alpha_{11} \frac{d M_{1}}{d \bar{r}}+\left(\lambda \alpha_{11}+\alpha_{12}\right) \frac{M_{1}}{\bar{r}}\right)+\alpha_{11} M_{1} \frac{d F_{1}}{d \bar{r}}\right\}+\bar{r}^{(-\lambda)}\left\{\left(C_{2}-F_{2}\right)\left(\alpha_{11} \frac{d M_{2}}{d \bar{r}}-\left(\lambda \alpha_{11}-\alpha_{12}\right) \frac{M_{2}}{\bar{r}}\right)-\alpha_{11} M_{2} \frac{d F_{2}}{d \bar{r}}\right\}\right] \\
& \sigma_{\theta \theta}(\bar{r})= \\
& \frac{1-n \bar{r}^{k}}{b}\left[\bar{r}^{(\lambda)}\left\{\left(C_{1}+F_{1}\right)\left(\alpha_{12} \frac{d M_{1}}{d \bar{r}}+\left(\lambda \alpha_{12}+\alpha_{22}\right) \frac{M_{1}}{\bar{r}}\right)+\alpha_{12} M_{1} \frac{d F_{1}}{d \bar{r}}\right\}+\bar{r}^{(-\lambda)}\left\{\left(C_{2}-F_{2}\right)\left(\alpha_{12} \frac{d M_{2}}{d \bar{r}}-\left(\lambda \alpha_{12}-\alpha_{22}\right) \frac{M_{2}}{\bar{r}}\right)-\alpha_{12} M_{2} \frac{d F_{2}}{d \bar{r}}\right\}\right] \\
& \sigma_{z z}(\bar{r})= \\
& \frac{1-n \bar{r}^{k}}{b}\left[\bar{r}^{(\lambda)}\left\{\left(C_{1}+F_{1}\right)\left(\alpha_{13} \frac{d M_{1}}{d \bar{r}}+\left(\lambda \alpha_{13}+\alpha_{23}\right) \frac{M_{1}}{\bar{r}}\right)+\alpha_{13} M_{1} \frac{d F_{1}}{d \bar{r}}\right\}+\bar{r}^{(-\lambda)}\left\{\left(C_{2}-F_{2}\right)\left(\alpha_{13} \frac{d M_{2}}{d \bar{r}}-\left(\lambda \alpha_{13}-\alpha_{23}\right) \frac{M_{2}}{\bar{r}}\right)-\alpha_{13} M_{2} \frac{d F_{2}}{d \bar{r}}\right\}\right] .
\end{aligned}
$$

Note that, if $n=m=0$ then $h(r)=h_{0}, c_{i j}=\alpha_{i j}, \rho=\rho_{0}$ and the radial displacement given in Equation (20) for the rotating uniform thickness and density homogeneous orthotropic hollow cylinder is reduced to

$$
\begin{aligned}
& F_{1}(\bar{r})=\frac{\rho_{0} \Omega^{2} b^{3}}{\alpha_{11}} \int_{0}^{\bar{r}} \frac{\varphi^{2-\lambda} e^{-m \varphi^{k}} M_{2}(\varphi)}{\left(1-n \varphi^{k}\right) F(\varphi)} d \varphi, \\
& F_{2}(\bar{r})=\frac{\rho_{0} \Omega^{2} b^{3}}{\alpha_{11}} \int_{0}^{\bar{r}} \frac{\varphi^{2+\lambda} e^{-m \varphi^{k}} M_{1}(\varphi)}{\left(1-n \varphi^{k}\right) F(\varphi)} d \varphi .
\end{aligned}
$$
yields the radial, circumferential and axial stresses for the rotating variable thickness and density inhomogeneous orthotropic hollow cylinder in the following form:

$$
\begin{aligned}
& \sigma_{r r}(\bar{r})=\frac{1}{b}\left[C_{1}\left(\lambda \alpha_{11}+\alpha_{12}\right) \bar{r}^{(\lambda-1)}-C_{2}\left(\lambda \alpha_{11}-\alpha_{12}\right) \bar{r}^{(-\lambda-1)}\right]+\frac{3 \alpha_{11}+\alpha_{12}}{\alpha_{11}\left(\lambda^{2}-9\right)} \rho_{0} \Omega^{2} b^{2} \bar{r}^{2} \\
& \sigma_{\theta \theta}(\bar{r})=\frac{1}{b}\left[C_{1}\left(\lambda \alpha_{11}+\alpha_{22}\right) \bar{r}^{(\lambda-1)}-C_{2}\left(\lambda \alpha_{12}-\alpha_{22}\right) \bar{r}^{(-\lambda-1)}\right]+\frac{3 \alpha_{12}+\alpha_{22}}{\alpha_{11}\left(\lambda^{2}-9\right)} \rho_{0} \Omega^{2} b^{2} \bar{r}^{2} \\
& \sigma_{z z}(\bar{r})=\frac{1}{b}\left[C_{1}\left(\lambda \alpha_{13}+\alpha_{23}\right) \bar{r}^{(\lambda-1)}-C_{2}\left(\lambda \alpha_{13}-\alpha_{23}\right) \bar{r}^{(-\lambda-1)}\right]+\frac{3 \alpha_{13}+\alpha_{23}}{\alpha_{11}\left(\lambda^{2}-9\right)} \rho_{0} \Omega^{2} b^{2} \bar{r}^{2}
\end{aligned}
$$

In addition, for isotropic cylinder we have [18]

$\alpha_{11}=\alpha_{22}=\frac{E(1-v)}{(1+v)(1-2 v)}, \quad \alpha_{12}=\alpha_{13}=\alpha_{23}=\frac{E v}{(1+v)(1-2 v)}$,

where $E$ and $v$ are Young's modulus and Poisson's ratio of the cylinder material. Using Equation (28) we find that the solution given in Equations (26) and (27) for the rotating uniform thickness and density homogeneous isotropic hollow cylinder takes the form:

$$
\begin{aligned}
& u(\bar{r})=C_{1} \bar{r}+\frac{C_{2}}{\bar{r}}-\frac{(1+v)(1-2 v)}{8 E(1-v)} \rho_{0} \Omega^{2} b^{3} \bar{r}^{3}, \\
& \sigma_{r r}(\bar{r})=\frac{E}{b(1+v)(1-2 v)}\left[C_{1}-C_{2} \frac{(1-2 v)}{\bar{r}^{2}}\right]-\frac{(3-2 v)}{8(1-v)} \rho_{0} \Omega^{2} b^{2} \bar{r}^{2}, \\
& \sigma_{\theta \theta}(\bar{r})=\frac{E}{b(1+v)(1-2 v)}\left[C_{1}+C_{2} \frac{(1-2 v)}{\bar{r}^{2}}\right]-\frac{(1+2 v)}{8(1-v)} \rho_{0} \Omega^{2} b^{2} \bar{r}^{2}, \\
& \sigma_{z z}(\bar{r})=\frac{2 E v}{b(1+v)(1-2 v)}\left[C_{1}-\frac{(1+v)(1-2 v)}{4 E(1-v)} \rho_{0} \Omega^{2} b^{3} \bar{r}^{2}\right] .
\end{aligned}
$$

also, the corresponding stresses in this case are given by: 
The previous elastic solutions will be completed by calculating the integration constants $C_{i}$ using various boundary conditions on the surfaces of the hollow cylinder.

\section{Rotation of Elastic Hollow Cylinders}

In the present section, we will obtain the elastic solutions for the rotating hollow cylinder. For the present hollow cylinder, the solution requires that one boundary condition be satisfied at each surface. The radial stress must be vanished at the free surface $(F)$ of the cylinder while the radial displacement must be equal to zero at the clamped surface (C) of the cylinder.

\subsection{Free-Free (FF) Hollow Cylinder}

When the inner and outer surfaces $(r=a, r=b$ or $\bar{r}=a / b=\bar{a}, \bar{r}=1$ ) of the cylinder are free of any traction, the boundary conditions are given by:

$$
\begin{array}{llll}
\sigma_{r r}(\bar{r})=0 & \text { at } & \bar{r}=\bar{a}, \\
\sigma_{r r}(\bar{r})=0 & \text { at } & \bar{r}=1 .
\end{array}
$$

Using the above conditions into Equation (23), the constants $C_{1}$ and $C_{2}$ are given by

$$
\begin{aligned}
& C_{1}=\frac{\left[F_{1}(1) S_{21}-F_{2}(1) S_{22}+S_{23}\right] S_{12}-\left[F_{1}(\bar{a}) S_{11}-F_{2}(\bar{a}) S_{12}+S_{13}\right] S_{22}}{S_{11} S_{22}-S_{12} S_{21}}, \\
& C_{2}=\frac{\left[F_{1}(\bar{a}) S_{11}-F_{2}(\bar{a}) S_{12}+S_{13}\right] S_{21}-\left[F_{1}(1) S_{21}-F_{2}(1) S_{22}+S_{23}\right] S_{11}}{S_{11} S_{22}-S_{12} S_{21}},
\end{aligned}
$$

where

$$
\begin{aligned}
& S_{11}=\bar{a}^{(\lambda)}\left[\alpha_{11} M_{1}^{\prime}(\bar{a})+\left(\lambda \alpha_{11}+\alpha_{12}\right) \frac{M_{1}(\bar{a})}{\bar{a}}\right] \\
& S_{12}=\bar{a}^{(-\lambda)}\left[\alpha_{11} M_{2}^{\prime}(\bar{a})-\left(\lambda \alpha_{11}-\alpha_{12}\right) \frac{M_{2}(\bar{a})}{\bar{a}}\right] \\
& S_{13}=\alpha_{11}\left[\bar{a}^{(\lambda)} M_{1}(\bar{a}) F_{1}^{\prime}(\bar{a})-\bar{a}^{(-\lambda)} M_{2}(\bar{a}) F_{2}^{\prime}(\bar{a})\right] \\
& S_{21}=\alpha_{11} M_{1}^{\prime}(1)+\left(\lambda \alpha_{11}+\alpha_{12}\right) M_{1}(1) \\
& S_{22}=\alpha_{11} M_{2}^{\prime}(1)-\left(\lambda \alpha_{11}-\alpha_{12}\right) M_{2}(1) \\
& S_{23}=\alpha_{11}\left[M_{1}(1) F_{1}^{\prime}(1)-M_{2}(1) F_{2}^{\prime}(1)\right]
\end{aligned}
$$

in which the prime $\left({ }^{\prime}\right)$ means differentiation with respect to $\bar{r}$.

The radial displacement and stresses for the rotating variable thickness and density inhomogeneous orthotropic hollow cylinder with free surfaces can be calculated from Equations (20), (23)-(25) and (32).

The solution given in Equations (26) and (27) for the rotating uniform thickness and density homogeneous orthotropic hollow cylinder with free surfaces can be obtained with the help of the following constants:

$$
\begin{aligned}
& C_{1}=\frac{\left(3 \alpha_{11}+\alpha_{12}\right)\left[\bar{a}^{(-\lambda-3)}-1\right] \bar{a}^{2} \rho_{0} \Omega^{2} b^{3}}{\alpha_{11}\left(\lambda^{2}-9\right)\left(\lambda \alpha_{11}+\alpha_{12}\right)\left[\bar{a}^{(\lambda-1)}-\bar{a}^{(-\lambda-1)}\right]}, \\
& C_{2}=\frac{\left(3 \alpha_{11}+\alpha_{12}\right)\left[\bar{a}^{(\lambda-3)}-1\right] \bar{a}^{2} \rho_{0} \Omega^{2} b^{3}}{\alpha_{11}\left(\lambda^{2}-9\right)\left(\lambda \alpha_{11}+\alpha_{12}\right)\left[\bar{a}^{(\lambda-1)}-\bar{a}^{(-\lambda-1)}\right]}
\end{aligned}
$$

Also, the radial displacement and stresses given in Equation (29) for the rotating uniform thickness and density homogeneous isotropic hollow cylinder with free surfaces can be written as

$$
\begin{aligned}
& u(\bar{r})=\frac{(1+v)(1-2 v)}{8 E(1-v)}\left[\left(1+\bar{a}^{2}\right)(3-2 v)+\frac{3-2 v}{1-2 v} \frac{\bar{a}^{2}}{\bar{r}^{2}}-\bar{r}^{2}\right] \rho_{0} \Omega^{2} b^{3} \bar{r} \\
& \sigma_{r r}(\bar{r})=\frac{3-2 v}{8(1-v)}\left[1+\bar{a}^{2}-\frac{\bar{a}^{2}}{\bar{r}^{2}}-\bar{r}^{2}\right] \rho_{0} \Omega^{2} b^{2} \\
& \sigma_{\theta \theta}(\bar{r})=\frac{3-2 v}{8(1-v)}\left[1+\bar{a}^{2}+\frac{\bar{a}^{2}}{\bar{r}^{2}}-\frac{1+2 v}{3-2 v} \bar{r}^{2}\right] \rho_{0} \Omega^{2} b^{2} \\
& \sigma_{z z}(\bar{r})=\frac{v}{4(1-v)}\left[\left(1+\bar{a}^{2}\right)(3-2 v)-2 \bar{r}^{2}\right] \rho_{0} \Omega^{2} b^{2}
\end{aligned}
$$


This is the well-known solution of the rotating uni- by: form thickness cylinder [19].

\subsection{Clamped-Clamped (CC) Hollow Cylinder}

When the inner and outer surfaces $(\bar{r}=\bar{a}, \bar{r}=1)$ of the cylinder are clamped, the boundary conditions are given

$$
\begin{aligned}
& C_{1}=\frac{\left[M_{1}(1) F_{1}(1)-M_{2}(1) F_{2}(1)\right] S_{41}-\left[F_{1}(\bar{a}) S_{14}-F_{2}(\bar{a}) S_{41}\right] M_{2}(1)}{M_{2}(1) S_{14}-M_{1}(1) S_{41}}, \\
& C_{2}=\frac{\left[F_{1}(\bar{a}) S_{14}-F_{2}(\bar{a}) S_{41}\right] M_{1}(1)-\left[M_{1}(1) F_{1}(1)-M_{2}(1) F_{2}(1)\right] S_{14}}{M_{2}(1) S_{14}-M_{1}(1) S_{41}},
\end{aligned}
$$

where

$$
S_{14}=\bar{a}^{(\lambda)} M_{1}(\bar{a}), \quad S_{41}=\bar{a}^{(-\lambda)} M_{2}(\bar{a}) .
$$

With the help of Equations (20), (23)-(25) and(36) ,we can obtain the radial displacement and stresses for the rotating variable thickness and density inhomogeneous orthotropic hollow cylinder with clamped surfaces.

The solution given in Equation (27) for the rotating uniform thickness and density homogeneous orthotropic hollow cylinder with clamped surfaces can be calculated with the aid of the following constants:

$$
C_{1}=\frac{\left[\bar{a}^{(-\lambda-3)}-1\right] \bar{a}^{3} \rho_{0} \Omega^{2} b^{3}}{\alpha_{11}\left(\lambda^{2}-9\right)\left[\bar{a}^{(\lambda)}-\bar{a}^{(-\lambda)}\right]}, \quad C_{2}=\frac{-\left[\bar{a}^{(\lambda-3)}-1\right] \bar{a}^{3} \rho_{0} \Omega^{2} b^{3}}{\alpha_{11}\left(\lambda^{2}-9\right)\left[\bar{a}^{(\lambda)}-\bar{a}^{(-\lambda)}\right]} .
$$

Finally, the radial displacement and stresses given in Equation (29) for the rotating uniform thickness and density homogeneous isotropic hollow cylinder with clamped surfaces becomes

$$
\begin{aligned}
& u(\bar{r})=\frac{(1+v)(1-2 v)}{8 E(1-v)}\left[1+\bar{a}^{2}-\frac{\bar{a}^{2}}{\bar{r}^{2}}-\bar{r}^{2}\right] \rho_{0} \Omega^{2} b^{3} \bar{r}, \\
& \sigma_{r r}(\bar{r})=\frac{\rho_{0} \Omega^{2} b^{2}}{8(1-v)}\left[1+\bar{a}^{2}+\frac{(1-2 v) \bar{a}^{2}}{\bar{r}^{2}}-(3-2 v) \bar{r}^{2}\right], \\
& \sigma_{\theta \theta}(\bar{r})=\frac{\rho_{0} \Omega^{2} b^{2}}{8(1-v)}\left[1+\bar{a}^{2}-\frac{(1-2 v) \bar{a}^{2}}{\bar{r}^{2}}-(1+2 v) \bar{r}^{2}\right], \\
& \sigma_{z z}(\bar{r})=\frac{v}{4(1-v)}\left[1+\bar{a}^{2}-2 \bar{r}^{2}\right] \rho_{0} \Omega^{2} b^{2} .
\end{aligned}
$$

\subsection{Free-Clamped (FC) Hollow Cylinder}

When the inner surface of the cylinder $C_{1}(\bar{r}=\bar{a})$ is free of any traction and the outer surface $(\bar{r}=1)$ is clamped, the boundary conditions are given by:

$$
\begin{aligned}
& \sigma_{r r}(\bar{r})=0 \quad \text { at } \quad \bar{r}=\bar{a}, \\
& u(\bar{r})=0 \quad \text { at } \quad \bar{r}=1 .
\end{aligned}
$$

From Equations (40), (20) and (23), the constants $C_{1}$ and $C_{2}$ are given by

$$
\begin{aligned}
& C_{1}=\frac{\left[M_{1}(1) F_{1}(1)-M_{2}(1) F_{2}(1)\right] S_{12}-\left[F_{1}(\bar{a}) S_{11}-F_{2}(\bar{a}) S_{12}+S_{13}\right] M_{2}(1)}{M_{2}(1) S_{11}-M_{1}(1) S_{12}}, \\
& C_{2}=\frac{\left[F_{1}(\bar{a}) S_{11}-F_{2}(\bar{a}) S_{12}+S_{13}\right] M_{1}(1)-\left[M_{1}(1) F_{1}(1)-M_{2}(1) F_{2}(1)\right] S_{11}}{M_{2}(1) S_{11}-M_{1}(1) S_{12}} .
\end{aligned}
$$

Substituting from these constants into Equations (20), (23)-(25), we can get the radial displacement and stresses for the rotating variable thickness and density inhomogeneous orthotropic hollow cylinder with free inner and clamped outer surfaces.
In addition, the solution for the rotating uniform thickness and density homogeneous orthotropic hollow cylinder with free inner and clamped outer surfaces can be obtained from Equations (26) and (27) with the help of the following constants: 


$$
\begin{aligned}
& C_{1}=\frac{-\left[\left(\lambda \alpha_{11}-\alpha_{12}\right) \bar{a}^{(-\lambda-3)}+\left(3 \alpha_{11}+\alpha_{12}\right)\right] \bar{a}^{2} \rho_{0} \Omega^{2} b^{3}}{\alpha_{11}\left(\lambda^{2}-9\right)\left[\left(\lambda \alpha_{11}+\alpha_{12}\right) \bar{a}^{(\lambda-1)}+\left(\lambda \alpha_{11}-\alpha_{12}\right) \bar{a}^{(-\lambda-1)}\right]} \\
& C_{2}=\frac{-\left[\left(\lambda \alpha_{11}+\alpha_{12}\right) \bar{a}^{(\lambda-3)}-\left(3 \alpha_{11}+\alpha_{12}\right)\right] \bar{a}^{2} \rho_{0} \Omega^{2} b^{3}}{\alpha_{11}\left(\lambda^{2}-9\right)\left[\left(\lambda \alpha_{11}+\alpha_{12}\right) \bar{a}^{(\lambda-1)}-\left(\lambda \alpha_{11}-\alpha_{12}\right) \bar{a}^{(-\lambda-1)}\right]} \\
& u(\bar{r})=\frac{(1+v)(1-2 v)}{8 E(1-v)}\left[\frac{1-2 v+\bar{a}^{4}(3-2 v)}{1-2 v+\bar{a}^{2}}+\frac{1-\bar{a}^{2}(3-2 v)}{1-2 v+\bar{a}^{2}} \frac{\bar{a}^{2}}{\bar{r}^{3}}-\bar{r}^{2}\right] \rho_{0} \Omega^{2} b^{3} \bar{r}, \\
& \sigma_{r r}(\bar{r})=\frac{\rho_{0} \Omega^{2} b^{2}}{8(1-v)}\left[\frac{1-2 v+\bar{a}^{4}(3-2 v)}{1-2 v+\bar{a}^{2}}-\frac{1-\bar{a}^{2}(3-2 v)}{1-2 v+\bar{a}^{2}} \frac{(1-2 v) \bar{a}^{2}}{\bar{r}^{2}}-(3-2 v) \bar{r}^{2}\right] \text {, } \\
& \sigma_{\theta \theta}(\bar{r})=\frac{\rho_{0} \Omega^{2} b^{2}}{8(1-v)}\left[\frac{1-2 v+\bar{a}^{4}(3-2 v)}{1-2 v+\bar{a}^{2}}+\frac{1-\bar{a}^{2}(3-2 v)}{1-2 v+\bar{a}^{2}} \frac{(1-2 v) \bar{a}^{2}}{\bar{r}^{2}}-(1+2 v) \bar{r}^{2}\right] \text {, } \\
& \sigma_{z z}(\bar{r})=\frac{v}{4(1-v)}\left[\frac{1-2 v+\bar{a}^{4}(3-2 v)}{1-2 v+\bar{a}^{2}}-2 \bar{r}^{2}\right] \rho_{0} \Omega^{2} b^{2} \text {. }
\end{aligned}
$$

Also, the radial displacement and stresses given in Equation (29) for the rotating uniform thickness and density homogeneous isotropic hollow cylinder with free inner and clamped outer surfaces can be obtained in the

\subsection{Clamped-Free (CF) Hollow Cylinder}

When the inner surface of the cylinder $(\bar{r}=\bar{a})$ is clamped and the outer surface $(\bar{r}=1)$ is free of any traction, the boundary conditions are given by:

$$
\begin{aligned}
& u(\bar{r})=0 \quad \text { at } \quad \bar{r}=\bar{a}, \\
& \sigma_{r r}(\bar{r})=0 \quad \text { at } \quad \bar{r}=1 .
\end{aligned}
$$

From Equations (20), (23) and (44), the constants $C_{1}$ and $C_{2}$ are given by

$$
\begin{aligned}
& C_{1}=\frac{\left[F_{1}(1) S_{21}-F_{2}(1) S_{22}+S_{23}\right] S_{41}-\left[F_{1}(\bar{a}) S_{14}-F_{2}(\bar{a}) S_{41}\right] S_{22}}{S_{22} S_{14}-S_{21} S_{41}}, \\
& C_{2}=\frac{\left[F_{1}(\bar{a}) S_{14}-F_{2}(\bar{a}) S_{41}\right] S_{21}-\left[F_{1}(1) S_{21}-F_{2}(1) S_{22}+S_{23}\right] S_{14}}{S_{22} S_{14}-S_{21} S_{41}}
\end{aligned}
$$

The radial displacement and stresses for the rotating variable thickness and density inhomogeneous orthotropic hollow cylinder with clamped inner and free outer surfaces can be obtained from Equations (20), (23)-(25) and (45).

Also, the solution given in Equation (27) for the rotating uniform thickness and density homogeneous orthotropic hollow cylinder with clamped inner and free outer surfaces can be calculated with the help of the following constants:

$$
\begin{aligned}
& C_{1}=\frac{-\left[\left(3 \alpha_{11}+\alpha_{12}\right) \bar{a}^{(-\lambda-3)}+\left(\lambda \alpha_{11}-\alpha_{12}\right)\right] \bar{a}^{3} \rho_{0} \Omega^{2} b^{3}}{\alpha_{11}\left(\lambda^{2}-9\right)\left[\left(\lambda \alpha_{11}-\alpha_{12}\right) \bar{a}^{(\lambda)}+\left(\lambda \alpha_{11}+\alpha_{12}\right) \bar{a}^{(-\lambda)}\right]}, \\
& C_{2}=\frac{\left[\left(3 \alpha_{11}+\alpha_{12}\right) \bar{a}^{(\lambda-3)}-\left(\lambda \alpha_{11}+\alpha_{12}\right)\right] \bar{a}^{3} \rho_{0} \Omega^{2} b^{3}}{\alpha_{11}\left(\lambda^{2}-9\right)\left[\left(\lambda \alpha_{11}-\alpha_{12}\right) \bar{a}^{(\lambda)}+\left(\lambda \alpha_{11}+\alpha_{12}\right) \bar{a}^{(-\lambda)}\right]} .
\end{aligned}
$$

Finally, one can obtain easily the radial displacement and stresses given in Equation (29) for the rotating uniform thickness and density homogeneous isotropic hollow cylinder with clamped inner and free outer surfaces in the following form:

$$
\begin{aligned}
& u(\bar{r})=\frac{(1+v)(1-2 v)}{8 E(1-v)}\left[\frac{3-2 v+\bar{a}^{4}(1-2 v)}{1-2 v+\bar{a}^{2}}-\frac{3-2 v-\bar{a}^{2} \bar{a}^{2}}{1-2 v+\bar{a}^{2}} \frac{\bar{r}^{3}}{\left.\bar{r}^{2}\right] \rho_{0} \Omega^{2} b^{3} \bar{r},}\right. \\
& \sigma_{r r}(\bar{r})=\frac{\rho_{0} \Omega^{2} b^{2}}{8(1-v)}\left[\frac{3-2 v+\bar{a}^{4}(1-2 v)}{1-2 v+\bar{a}^{2}}+\frac{3-2 v-\bar{a}^{2}}{1-2 v+\bar{a}^{2}} \frac{(1-2 v) \bar{a}^{2}}{\bar{r}^{2}}-(3-2 v) \bar{r}^{2}\right], \\
& \sigma_{\theta \theta}(\bar{r})=\frac{\rho_{0} \Omega^{2} b^{2}}{8(1-v)}\left[\frac{3-2 v+\bar{a}^{4}(1-2 v)}{1-2 v+\bar{a}^{2}}-\frac{3-2 v-\bar{a}^{2}}{1-2 v+\bar{a}^{2}} \frac{(1-2 v) \bar{a}^{2}}{\bar{r}^{2}}-(1+2 v) \bar{r}^{2}\right], \\
& \sigma_{z z}(\bar{r})=\frac{v}{4(1-v)}\left[\frac{3-2 v+\bar{a}^{4}(1-2 v)}{1-2 v+\bar{a}^{2}}-2 \bar{r}^{2}\right] \rho_{0} \Omega^{2} b^{2} .
\end{aligned}
$$




\section{Conclusions}

The rotation problem of a variable-thickness inhomogeneous, orthotropic, hollow cylinder has been studied. Analytical solution for rotating variable-thickness, inhomogeneous, orthotropic, hollow cylinder subjected to different boundary conditions are derived. The displacement and stresses for rotating uniform-thickness, homogeneous, isotropic, hollow cylinder are obtained as special cases of the investigated problem. In the second part of this paper we will present the corresponding viscoelastic solutions and some applications concerning the effects due to many parameters on the radial displacement and stresses.

\section{References}

[1] Yu. É. Senitskii, "Stress State of a Rotating Inhomogeneous Anisotropic Cylinder of Variable Density,” International Applied Mechanics, Vol. 28, No. 5, 1992, pp. 28-35.

[2] C. O. Horgan and A. M. Chan, "The Pressurized Hollow Cylinder or Disk Problem for Functionally Graded Isotropic Linear Elastic Materials,” Journal of Elasticity, Vol. 55, No. 1, 1999, pp. 43-59.

[3] A. T. Vasilenko and N. I. Klimenko, "Stress State of Rotating Inhomogeneous Anisotropic Cylinders," International Applied Mechanics, Vol. 35, No. 8, 1999, pp. 778-783.

[4] F. Rooney and M. Ferrari, "Tension, Bending, and Flexure of Functionally Graded Cylinders,” International Journal of Solids and Structures, Vol. 38, No. 3, 2001, pp. 413-421.

[5] Ya. M. Grigorenko and A. T. Vasilenko, "The Effect of Inhomogeneity of Elastic Properties on the Stress State in Composite Cylindrical Panels,” Mechanics of Composite Materials, Vol. 37, No. 2, 2001, pp. 85-90.

[6] A. Oral and G. Anlas, "Effects of Radially Varying Moduli on Stress Distribution of Nonhomogeneous Anisotropic Cylindrical Bodies,” International Journal of Solids and Structures, Vol. 42, No. 20, 2005, pp. 5568-5588.

[7] E. Pan and A. K. Roy, "A Simple Plane-strain Solution for Functionally Graded Multilayered Isotropic Cylinders," Structural Engineering and Mechanics, Vol. 24, No. 6, 2006, pp. 727-740.
[8] N. Tutuncu, "Stresses in Thick-walled FGM Cylinders with Exponentially-varying Properties," Engineering Structures, Vol. 29, No. 9, 2007, pp. 2032-2035.

[9] E. E. Theotokoglou and I. H. Stampouloglou, "The Radially Nonhomogeneous Elastic Axisymmentric Problem,” International Journal of Solids and Structures, Vol. 45, No. 25-26, 2008, pp. 6535-6552.

[10] M. Mohammadi and J. R. Dryden, "Influence of the Spatial Variation of Poisson's Ratio upon the Elastic Field in Nonhomogeneous Axisymmetric Bodies,” International Journal of Solids and Structures, Vol. 46, No. 3-4, 2009, pp. 788-795.

[11] X. F. Li and X. L. Peng, "A Pressurized Functionally Graded Hollow Cylinder with Arbitrarily Varying Material Properties,” Journal of Elasticity, Vol. 96, No. 1, 2009, pp. 81-95.

[12] Ya. M. Grigorenko and L. S. Rozhok, "Stress Analysis of Hollow Elliptic Cylinders with Variable Eccentricity and Thickness,” International Applied Mechanics, Vol. 38, No. 8, 2002, pp. 954-966.

[13] A. M. Zenkour, "Rotating Variable-thickness Orthotropic Cylinder Containing a Solid Core of Uniform-thickness," Archive of Applied Mechanics, Vol. 76, No. 1-2, 2006, pp. 89-102.

[14] A. M. Zenkour, "Stresses in Cross-ply Laminated Circular Cylinders of Axially Variable Thickness," Acta Mechanica, Vol. 187, No. 1-4, 2006, pp. 85-102.

[15] W. H. Duan and C. G. Koh, "Axisymmetric Transverse Vibrations of Circular Cylindrical Shells with Variable Thickness,” Journal of Sound and Vibration, Vol. 317, No. 3-5, 2008, pp. 1035-1041.

[16] G. J. Nie and R. C. Batra , "Static Deformations of Functionally Graded Polar-orthotropic Cylinders with Elliptical Inner and Circular Outer Surfaces," Composites Science and Technology, Vol. 70, No. 3, 2010, pp. 450-457.

[17] M. Abramowitz and A. I. Stegun, "Handbook of Mathematical Functions,” 5th Edition, US Government Printing Office, Washington DC, 1966.

[18] H. J. Ding, H. M. Wang and W.Q. Chen, "A Solution of a Non-homogeneous Orthotropic Cylindrical Shell for Axisymmetric Plane Strain Dynamic Thermoelastic Problems,” Journal of Sound and Vibration, Vol. 236, No. 4, 2003, pp. 815-829.

[19] L. S. Srinath, "Advanced Mechanics of Solids," McGraw-Hill, India, 1983. 